\title{
The relation between air pollution and respiratory deaths in Tehran, Iran- using generalized additive models
}

\author{
Azizallah Dehghan ${ }^{1}$, Narges Khanjani ${ }^{2,3,9^{*}}$, Abbas Bahrampour ${ }^{4}$, Gholamreza Goudarzi, ${ }^{5,6}$ and Masoud Yunesian ${ }^{7,8}$
}

\begin{abstract}
Background: Some epidemiological evidence has shown a relation between ambient air pollution and adverse health outcomes. The aim of this study was to investigate the effect of air pollution on mortality from respiratory diseases in Tehran, Iran.
\end{abstract}

Methods: In this ecological study, air pollution data was inquired from the Tehran Province Environmental Protection Agency and the Tehran Air Quality Control Company. Meteorological data was collected from the Tehran Meteorology Organization and mortality data from the Tehran Cemetery Mortality Registration. Generalized Additive Models (GAM) was used for data analysis with different lags, up to 15 days. A 10-unit increase in all pollutants except CO (1-unit) was used to compute the Relative Risk of deaths.

Results: During 2005 until 2014, 37,967 respiratory deaths occurred in Tehran in which 21,913 (57.7\%) were male. The strongest relationship between $\mathrm{NO}_{2}$ and $\mathrm{PM}_{10}$ and respiratory death was seen on the same day (lag 0 ), and was respectively ( $R R=1.04,95 \% \mathrm{Cl}: 1.02-1.07)$ and $(R R=1.03,95 \% \mathrm{Cl}: 1.02-1.04) . \mathrm{O}_{3}$ and $\mathrm{PM}_{2.5}$ had the strongest relationship with respiratory deaths on lag 2 and 1 respectively, and the RR was equal to $1.03,95 \%$ Cl: $1.01-1$. 05 and 1.06, 95\% Cl: 1.02-1.10 respectively. $\mathrm{NO}_{2}, \mathrm{O}_{3}, \mathrm{PM}_{10}$ and $\mathrm{PM}_{2.5}$ also showed significant relations with respiratory deaths in the older age groups.

Conclusions: The findings of this study showed that $\mathrm{O}_{3}, \mathrm{NO}_{2}, \mathrm{PM}_{10}$ and $\mathrm{PM}_{2.5}$ air pollutants were related to respiratory deaths in Tehran. Reducing ambient air pollution can save lives in Tehran.

Keywords: Air pollution, Death, Respiratory disease

\section{Background}

Outdoor or indoor air pollution is caused by chemical, physical, and biological agents that change the normal composition of atmosphere. Household heating services, motor vehicles, industrial equipment, and forest fires are the most common sources of air pollution [1]. Nowadays air pollution is a complicated environmental health problem especially in developing countries [2]. Epidemiological evidence has shown relations between outdoor air pollution and various undesirable health consequences [2-5]. There are different ambient air

\footnotetext{
* Correspondence: n_khanjani@kmu.ac.ir

${ }^{2}$ Environmental Health Engineering Research Center, Kerman University of Medical Sciences, Kerman, Iran

${ }^{3}$ Monash Centre for Occupational \& Environmental Health, School of Public Health and Preventive Medicine, Monash University, Melbourne, Australia

Full list of author information is available at the end of the article
}

pollutants which affect human health in different ways. The most important pollutants are particulate matter (PM), sulfur dioxide $\left(\mathrm{SO}_{2}\right)$, nitrogen oxides $\left(\mathrm{NO}_{\mathrm{x}}\right)$, ozone $\left(\mathrm{O}_{3}\right)$, and carbon monoxide $(\mathrm{CO})[6]$.

Studies from Europe and America have showed that even short-term changes in air pollution can increase daily mortality $[4,5]$. Although the relative risk of mortality caused by air pollution is low, the proportion of deaths related to air pollution is high because of the high number of at-risk and sensitive populations [7].

It is estimated that outdoor air pollution in urban and rural areas caused 3 million early deaths around the world in 2012 and $88 \%$ of these deaths occur in low and middle-income countries, mostly in south East Asia and the western Pacific. According to the WHO estimations, 
$14 \%$ of early respiratory deaths and $14 \%$ of lung cancers are probably caused by air pollution [8].

Several studies have been done around the world about air pollutants and human deaths. However, most of them were done in developed countries and few studies were conducted in the Eastern Mediterranean region [9].

Air pollution is one of the most important environmental health problems in Tehran, the capital of Iran which has a population of over 12 million people. Controlling air pollution has been complicated in this city and investigation about the different aspects of air pollution and its health effects is still necessary $[10,11]$. In this paper, the impact of air pollutants on respiratory disease mortality in Tehran has been evaluated.

\section{Methods}

\section{Study design and setting}

This ecological and population based study was conducted to determine the relation between air pollution and respiratory deaths in Tehran, Iran in 2005-2014. Tehran is the largest city and capital of Iran with a population of over 12 million people.

\section{Air pollution, meteorological and death data}

Concentrations of ambient air pollutants were collected from the Tehran Air Quality Control Company and the Tehran Province Environment Protection Agency. There are 36 air pollution monitoring stations in Tehran, of which 21 operate under the Tehran Air Quality Control Company and the others are managed by the Tehran Province Environment Protection Agency. Tehran has 22 municipality districts and there are one or more air pollution monitoring stations in each district. Only 7 stations had data for 10 years under study. Other stations were between 1 to 5 years old. For this reason, we used the average daily pollutants in these seven stations as an indicator of air pollution in the whole city. These stations record ambient air pollutant concentrations per hour. We used average daily values in the analysis. We estimated missing data by the Expectation- Maximization algorithm (EM).

Meteorological data including temperature and relative humidity which are possible confounding parameters were collected from the Tehran Meteorological Organization. We used the average of daily temperature and relative humidity in this analysis. We had no missing meteorological data.

Data about number of mortalities caused by respiratory diseases in days were collected from the Tehran cemetery (Behesht-e-Zahra) and according to the international classification of diseases (ICD10) with J00-J99 codes (all respiratory deaths). We used aggregated death data for the whole city.

\section{Statistical analysis}

Descriptive statistics including mean, standard deviation, the 25th, 50th (median), 75th and 98th percentiles, minimum and maximum were computed by SPSS 16 .

Then the multi-pollutant Generalized Additive Model (GAM) was used for estimating the Rate Ratio (RR) of air pollutants' effect on respiratory mortality. This model is an expanded form of the Generalized Linear Model (GLM) and has high flexibility. This model has been used in many studies because it can adjust for nonlinear confounding parameters such as seasonal changes, trends and the weather variables $[12,13]$.

$$
\begin{aligned}
\mathrm{Y}_{\mathrm{t}} \sim \operatorname{Poisson}\left(\mu_{\mathrm{t}}\right) & \\
\log \mu_{\mathrm{t}}=\alpha & +\sum_{\text {day of week }} \beta_{\mathrm{i}}\left(\mathrm{X}_{\mathrm{i}}\right)+\sum \mathrm{S}_{\mathrm{j}}\left(\mathrm{X}_{\mathrm{j}}\right)+\text { season } \\
& + \text { da }
\end{aligned}
$$

where $Y_{t}$ denotes the daily number of deaths due to respiratory disease, $\beta \mathrm{i}$ is the coefficients for air pollutants (Xi) and denotes the log Relative Rate of death related with each 1 unit increase in $\mathrm{CO}$ and each 10 unit increase in all other pollutants; and $\mathrm{Sj}(\mathrm{Xj})$ are the smoothing functions of meteorological variables (temperature and relative humidity) [14]. The degree of freedom (df) for the smoothers is determined with Generalized Cross Validation (GCV) by using the mgcv package in $\mathrm{R}$ software.

In this study multi-pollutant models were used and pollutants with less than 0.2 correlation with the main predicting pollutant were entered into the model. All of the pollutants did not come into the model together, due to the high correlation between some pollutants.

In this part of the study, meteorology variables (humidity, temperature), season and day of the week were added to the model as potential confounders and their effects were adjusted by using smooth functions to determine the exact effects of pollutants on number of deaths. The time unit used in the analysis was day.

Using the formulas below, we computed the Relative Risk (RR) and 95\% Confidence Interval (CI) for RR.

$$
\begin{aligned}
& \mathrm{RR}=\exp (\beta) \\
& 95 \% \mathrm{CI}=\exp (\beta \pm 1.96 \mathrm{SE})
\end{aligned}
$$

Microsoft Office Excel 2007 and SPSS 16 software were used to prepare the data, and the ' $m g c v$ ' package in $\mathrm{R}$ i386 3.2.2 software was used for GAM analysis.

\section{Results}

In the 10-year period under study, 37,967 Respiratory deaths occurred in Tehran, in which 21,913 (57.73\%) cases were male and $16,047(42.27)$ were female. The male to female sex ratio was 1.36, 9065 (23.87\%) deaths 
occurred in spring, 8528 (22.46\%) in summer, 9323 (24.55\%) in autumn and 11,051 (29.1\%) in winter.

The frequency of male, female and total respiratory deaths in 2005-2014 are shown in Table 1.

In this study $13.1 \%$ of air pollution data was missing. The missing data was $10.51 \%$ in $\mathrm{O} 3,16.37 \%$ in $\mathrm{CO}$, $8.73 \%$ in $\mathrm{NO}_{2}, 10.51 \%$ in $\mathrm{SO}_{2}, 7.09 \%$ in $\mathrm{PM}_{10}$ and $35.10 \%$ in $\mathrm{PM}_{2.5}$. They were estimated using the EM algorithm method.

The descriptive statistics of air pollution data, meteorological data and respiratory deaths are shown in Table 2.

The result of this study showed that the daily average concentrations for $\mathrm{PM}_{2.5}$ was $20.68 \mu \mathrm{g} / \mathrm{m} 3$ and for $\mathrm{PM}_{10}$ was $69.75 \mu \mathrm{g} / \mathrm{m} 3$ and these two concentrations were higher than the WHO 2014 guideline annual thresholds (20 and $10 \mu \mathrm{g} / \mathrm{m}^{3}$, respectively). However, the 98th percentile of $\mathrm{CO}, \mathrm{O}_{3}$ and $\mathrm{SO}_{2}$ was less than the WHO threshold.

In 3173 from 3652 days, the $\mathrm{PM}_{10}$ concentration was higher than the WHO guideline daily threshold $(50 \mu \mathrm{g} /$ $\mathrm{m}^{3}$ ). Also in 315 days from 1826 days, the $\mathrm{PM}_{2.5}$ concentrations were higher than WHO guideline daily threshold $\left(25 \mu \mathrm{g} / \mathrm{m}^{3}\right)$.

The annual average of $\mathrm{NO}_{2}$ in 2004 to 2009 was higher, but in 2010 to 2013 was lower than WHO guidelines. The 75 percentile in all years was higher than WHO guidelines (50 ppb). Figure 1 shows the boxplots for all pollutants.

Table 3 shows the results of the adjusted Generalized Additive Model (multi-pollutant), about the effect of air pollutants on overall respiratory deaths. The table shows that ambient $\mathrm{O}_{3}$, and $\mathrm{NO}_{2}, \mathrm{PM}_{10}$ and $\mathrm{PM}_{2.5}$ may affect respiratory death. The strongest relationships for each of these pollutants were in lag 2 for $\mathrm{O}_{3}(\mathrm{RR}=1.03,95 \% \mathrm{CI}$; 1.01-1.05), lag 0 for $\mathrm{NO}_{2}(\mathrm{RR}=1.04,95 \% \mathrm{CI} ; 1.02-1.07)$, lag 0 for $\mathrm{PM}_{10}(\mathrm{RR}=1.03,95 \% \mathrm{CI} ; 1.02-1.04)$ and lag 1 for $\mathrm{PM}_{2.5}(\mathrm{RR}=1.06,95 \% \mathrm{CI} ; 1.02-1.10)$.

Tables 4 and 5 shows the effect of air pollutants on male and female respiratory deaths. Table 4 shows that $\mathrm{O}_{3}, \mathrm{NO}_{2}, \mathrm{PM}_{10}$ and $\mathrm{PM}_{2.5}$ had a direct association with respiratory deaths in males and females. In men, the strongest relation was seen in lag 0 for $\mathrm{O}_{3}(\mathrm{RR}=1.03$, 95\% CI; 1.00-1.06), in lag 0 for $\mathrm{PM}_{10}(\mathrm{RR}=1.03,95 \% \mathrm{CI}$; $1.02-1.05)$, in lag 1 for $\mathrm{NO}_{2}(\mathrm{RR}=1.04,95 \% \mathrm{CI}$; $1.00-1.06)$, and in lag 1 for $\mathrm{PM}_{2.5}(\mathrm{RR}=1.04,95 \% \mathrm{CI}$;
1.02-1.07). In women, only $\mathrm{NO}_{2}, \mathrm{PM}_{10}$ and $\mathrm{PM}_{2.5}$ were related with respiratory death. $\mathrm{NO}_{2}$ and $\mathrm{PM}_{10}$ had the highest relation with respiratory deaths at lag 0 and $\mathrm{PM}_{2.5}$ at lag 1 in women. The Relative Risk of respiratory deaths for $\mathrm{NO}_{2}, \mathrm{PM}_{10}$ and $\mathrm{PM}_{2.5}$ was $(\mathrm{RR}=$ 1.06, 95\% CI; 1.02-1.10), (RR =1.03, 95\% CI; 1.02-1.04) and $(\mathrm{RR}=1.05,95 \% \mathrm{CI} ; 1.01-1.09)$ respectively.

Tables 6, 7 and 8 show the exposure-response relation between air pollutants and respiratory deaths in age groups under 18, 18-60 and over 60 years. None of the air pollutants were related with respiratory deaths in people under 18 years old. But in the 18 to 60 and over 60 years old age group, $\mathrm{NO}_{2}, \mathrm{O}_{3}, \mathrm{PM}_{10}$ and $\mathrm{PM}_{2.5}$ showed significant relations. RRs can be seen in Tables 6, 7 and 8.

In men, women and in all age groups $\mathrm{CO}$ and $\mathrm{SO}_{2}$ showed no direct relation with respiratory disease. $\mathrm{SO}_{2}$ was not related to increase respiratory mortality, maybe because it did not exceed WHO thresholds except on 6 days in 10 years.

In this study, in some situations, $\mathrm{SO}_{2}$ showed an inverse relationship with respiratory death. More investigation about the probable harvesting effect of $\mathrm{SO}_{2}$ was done according to the methods suggested by previous studies [15]. The average count of deaths in the previous 14 days (two weeks) was divided by the average counts of deaths 3 days after exposure to $\mathrm{SO}_{2}$ and the ratio was 1.02 for all deaths, 1.03 for males and females. The RR for $\mathrm{SO}_{2}$ after adjusting for day of the week, season, meteorological variables and auto-correlation was 1.02 (95\% CI 1.01-1.03) and this auto-correlation was significant $(p<0.001)$ and shows the presence of harvesting. Also, the RR for death in percentiles above the 95th percentile compared to the 5th percentile was 1.11 and shows that death is higher in higher concentrations of $\mathrm{SO}_{2}$ which is rational.

\section{Discussion}

Air pollution, as one of the most important environmental health problems is a major threat to human health and leads to many adverse health effects, the most important of which is on the respiratory system. Studies have been carried out on its acute and chronic effects on the respiratory system [16]. The aim of this study was to investigate the relationship between respiratory mortality and air pollution.

Table 1 Frequency of male, female and total respiratory deaths in 2005-2014

\begin{tabular}{llllllllllll}
\hline & 2005 & 2006 & 2007 & 2008 & 2009 & 2010 & 2011 & 2012 & 2013 & 2014 & Total \\
\hline Male & 1772 & 1731 & 1774 & 1873 & 1850 & 2257 & 2323 & 2511 & 2936 & 2886 & 21,913 \\
Female & 1272 & 1290 & 1302 & 1210 & 1415 & 1700 & 1763 & 1803 & 2126 & 2166 & 16,047 \\
Sex Ratio & 1.39 & 1.34 & 1.36 & 1.54 & 1.30 & 1.32 & 1.31 & 1.39 & 1.38 & 1.33 & 1.365 \\
Age- standardized rate $^{\mathrm{a}}$ & 117.49 & 114.09 & 117.05 & 112.78 & 119.91 & 120.62 & 118.99 & 113.73 & 119.6 & 119.2 & - \\
\hline
\end{tabular}

${ }^{\mathrm{a}}$ Deaths per 100,000 population 
Table 2 Descriptive statistics of daily air pollution, meteorological data and mortality

\begin{tabular}{|c|c|c|c|c|c|c|c|c|}
\hline & Mean & SD & Min & 25th Percentile & Median & 75th percentile & 98th percentile & Max \\
\hline $\mathrm{O}_{3}(\mathrm{ppb})$ & 24.66 & 9.125 & 6.89 & 18.31 & 23.30 & 28.98 & 48.70 & 77.09 \\
\hline $\mathrm{CO}(\mathrm{ppm})$ & 3.29 & 0.97 & 1.47 & 2.61 & 3.13 & 3.81 & 5.81 & 11.29 \\
\hline $\mathrm{NO}_{2}(\mathrm{ppb})$ & 53.47 & 16.52 & 12.06 & 43.03 & 49.82 & 60.33 & 97.04 & 153.57 \\
\hline $\mathrm{SO}_{2}(\mathrm{ppb})$ & 33.46 & 10.47 & 9.02 & 26.87 & 32.05 & 38.26 & 62.08 & 119.10 \\
\hline $\mathrm{PM}_{10}\left(\mu \mathrm{g} / \mathrm{m}^{3}\right)$ & 69.75 & 20.61 & 12.02 & 57.39 & 68.04 & 80.65 & 117.2 & 331.20 \\
\hline $\mathrm{PM}_{2.5}\left(\mu \mathrm{g} / \mathrm{m}^{3}\right)$ & 20.68 & 5.87 & 7.43 & 16.72 & 20.23 & 23.67 & 35.85 & 55.06 \\
\hline Temperature $\left({ }^{\circ} \mathrm{C}\right)$ & 16.49 & 9.52 & -8.70 & 8.30 & 16.80 & 25.20 & 31.60 & 34.40 \\
\hline Relative humidity (\%) & 43.72 & 18.37 & 9.75 & 29.63 & 39.50 & 55.75 & 88.62 & 89 \\
\hline Male deaths(N) & 6.00 & 2.44 & 0 & 4 & 6 & 8 & 12 & 16 \\
\hline Female deaths $(\mathrm{N})$ & 4.39 & 1.90 & 0 & 3 & 4 & 6 & 9 & 16 \\
\hline Total deaths(N) & 10.40 & 3.97 & 2 & 7 & 10 & 13 & 19 & 31 \\
\hline
\end{tabular}

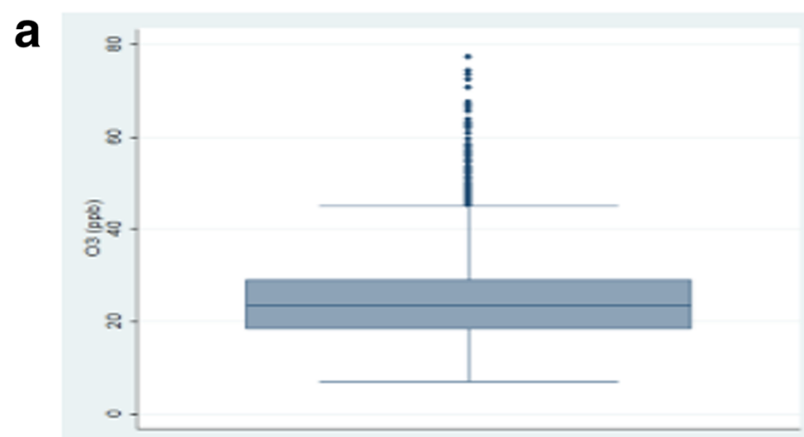

b

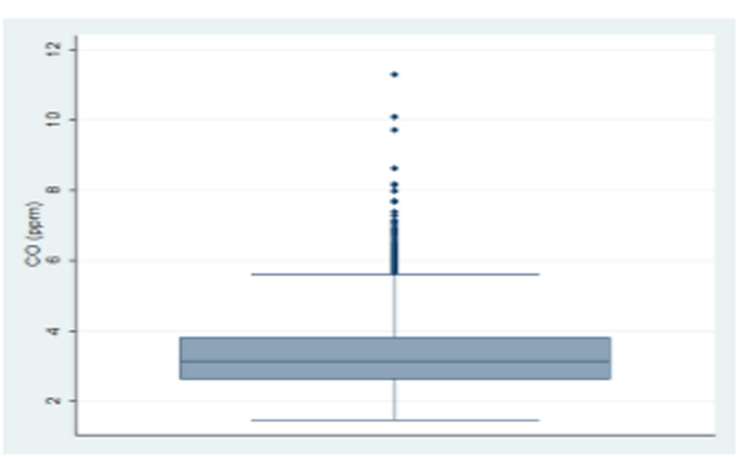

C

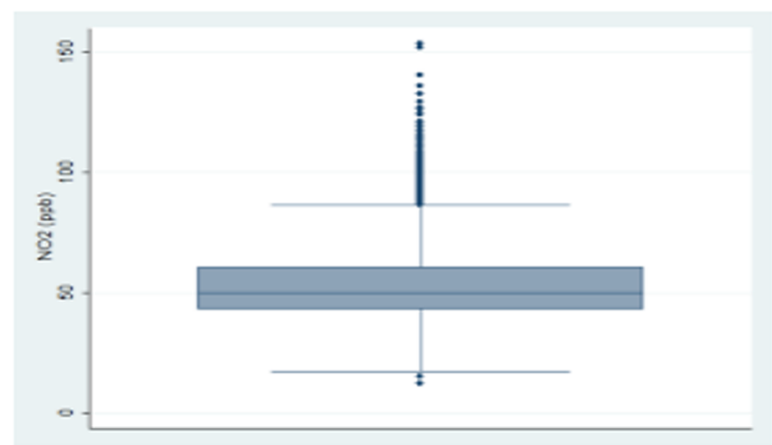

d

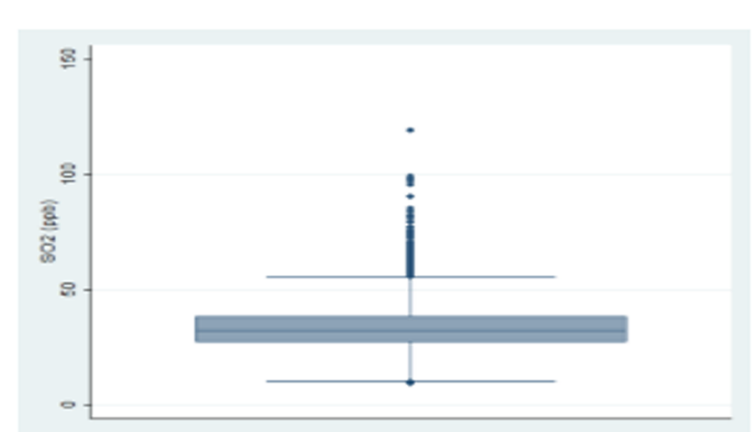

e

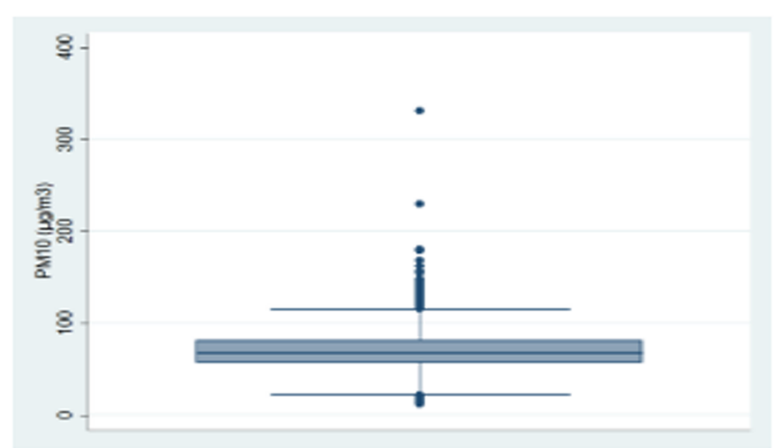

f

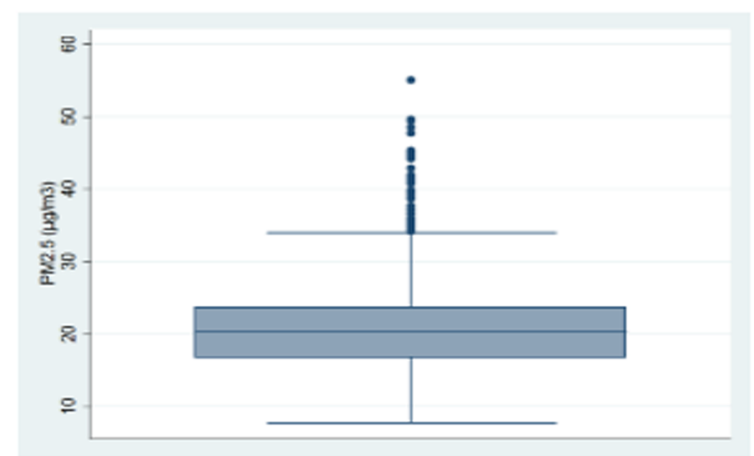

Fig. 1 The box plots for air pollutants $\left(\mathbf{a}: \mathrm{O}_{3}, \mathbf{b}: \mathrm{CO}, \mathbf{c}: \mathrm{NO}_{2}, \mathbf{d}: \mathrm{SO}_{2}, \mathbf{e}: \mathrm{PM}_{10}\right.$ and $\left.\mathbf{f}: \mathrm{PM}_{2.5}\right)$ 
Table 3 Results of adjusted Generalized Additive Model, about the effect of air pollutants on total respiratory deaths (for 1 unit increase in $\mathrm{CO}$ and 10 units increase in all other pollutants)

\begin{tabular}{|c|c|c|c|c|c|}
\hline & Lag & $d f$ & $\mathrm{RR}$ & $95 \% \mathrm{Cl}$ for $\mathrm{RR}$ & $P$-Value \\
\hline \multirow[t]{6}{*}{ Lag 0} & $\mathrm{O}_{3}(\mathrm{ppb})$ & 7.94 & 1.02 & 0.99-1.05 & 0.053 \\
\hline & $\mathrm{CO}$ (ppm) & 5.04 & 1.02 & $0.99-1.05$ & 0.139 \\
\hline & $\mathrm{NO}_{2}(\mathrm{ppb})$ & 3.04 & 1.04 & $1.02-1.07$ & $<0.001$ \\
\hline & $\mathrm{SO}_{2}(\mathrm{ppb})$ & 2.61 & 0.96 & $0.93-0.98$ & 0.006 \\
\hline & $\mathrm{PM}_{10}\left(\mu \mathrm{g} / \mathrm{m}^{3}\right)$ & 7.3 & 1.03 & $1.02-1.04$ & $<0.001$ \\
\hline & $\mathrm{PM}_{2.5}\left(\mu \mathrm{g} / \mathrm{m}^{3}\right)$ & 3.26 & 1.04 & $1.00-1.08$ & 0.008 \\
\hline \multirow[t]{6}{*}{ Lag 1} & $\mathrm{O}_{3}(\mathrm{ppb})$ & 4.04 & 1.03 & $1.00-1.05$ & 0.042 \\
\hline & CO (ppm) & 6.35 & 0.96 & $0.99-1.05$ & 0.130 \\
\hline & $\mathrm{NO}_{2}(\mathrm{ppb})$ & 6.51 & 1.04 & $1.01-1.06$ & 0.003 \\
\hline & $\mathrm{SO}_{2}(\mathrm{ppb})$ & 3.65 & 0.95 & $0.92-0.98$ & 0.004 \\
\hline & $\mathrm{PM}_{10}\left(\mu \mathrm{g} / \mathrm{m}^{3}\right)$ & 6.66 & 1.02 & $1.01-1.03$ & $<0.001$ \\
\hline & $\mathrm{PM}_{2.5}\left(\mu \mathrm{g} / \mathrm{m}^{3}\right)$ & 2.69 & 1.06 & $1.02-1.10$ & $<0.001$ \\
\hline \multirow[t]{6}{*}{ Lag 2} & $\mathrm{O}_{3}(\mathrm{ppb})$ & 5.34 & 1.03 & $1.01-1.06$ & 0.015 \\
\hline & CO (ppm) & 5.79 & 1.03 & 0.99-1.06 & 0.199 \\
\hline & $\mathrm{NO}_{2}(\mathrm{ppb})$ & 4.17 & 1.04 & $1.01-1.06$ & 0.001 \\
\hline & $\mathrm{SO}_{2}(\mathrm{ppb})$ & 4.21 & 0.95 & $0.92-0.98$ & 0.004 \\
\hline & $\mathrm{PM}_{10}\left(\mu \mathrm{g} / \mathrm{m}^{3}\right)$ & 6.79 & 1.02 & $1.01-1.03$ & $<0.001$ \\
\hline & $\mathrm{PM}_{2.5}\left(\mu \mathrm{g} / \mathrm{m}^{3}\right)$ & 2.41 & 1.04 & $1.00-1.08$ & 0.001 \\
\hline \multirow[t]{6}{*}{ Lag 3} & $\mathrm{O}_{3}(\mathrm{ppb})$ & 4.86 & 1.03 & $1.01-1.06$ & 0.015 \\
\hline & $\mathrm{CO}$ (ppm) & 5.08 & 1.02 & $0.99-1.05$ & 0.228 \\
\hline & $\mathrm{NO}_{2}(\mathrm{ppb})$ & 3.04 & 1.04 & $1.01-1.06$ & 0.001 \\
\hline & $\mathrm{SO}_{2}(\mathrm{ppb})$ & 8.14 & 0.95 & $0.92-0.98$ & 0.004 \\
\hline & $\mathrm{PM}_{10}\left(\mu \mathrm{g} / \mathrm{m}^{3}\right)$ & 6.44 & 1.02 & $1.01-1.03$ & 0.001 \\
\hline & $\mathrm{PM}_{2.5}\left(\mu \mathrm{g} / \mathrm{m}^{3}\right)$ & 2.56 & 1.03 & $0.98-1.07$ & 0.064 \\
\hline \multirow{6}{*}{$\begin{array}{l}\text { Mean } \\
\text { Lag 4-8 }\end{array}$} & $\mathrm{O}_{3}(\mathrm{ppb})$ & 3.42 & 1.02 & 0.99-1.05 & 0.103 \\
\hline & $\mathrm{CO}$ (ppm) & 6.51 & 1.02 & $0.98-1.04$ & 0.176 \\
\hline & $\mathrm{NO}_{2}(\mathrm{ppb})$ & 5.79 & 1.03 & $1.01-1.06$ & 0.004 \\
\hline & $\mathrm{SO}_{2}(\mathrm{ppb})$ & 4.83 & 0.95 & $0.93-0.99$ & 0.014 \\
\hline & $\mathrm{PM}_{10}\left(\mu \mathrm{g} / \mathrm{m}^{3}\right)$ & 6.94 & 1.01 & $1.00-1.02$ & 0.002 \\
\hline & $\mathrm{PM}_{2.5}\left(\mu \mathrm{g} / \mathrm{m}^{3}\right)$ & 3.08 & 1.01 & $0.97-1.05$ & 0.193 \\
\hline
\end{tabular}

From 2005 to 2014, the number of respiratory mortality in both men and women has gradually increased in Tehran. As the corresponding increase in AgeStandardized Rates was negligible, we can conclude that this increase was nearly entirely due to changes in population, such as increase in the urban population of Tehran due to immigration. However, this population increase is less likely to have affected our results. In time series regression studies, the denominator (the underlying population size) is not part of the dataset, and is not a concern because in these models, we are usually interested in modeling variation in outcome from day to day or week to week, and population size is unlikely to change meaningfully over these timescales, and can be
Table 4 Results of adjusted Generalized Additive Model and the effect of pollutants on respiratory deaths in men (for 1 unit increase in $\mathrm{CO}$ and 10 units increase in all other pollutants)

\begin{tabular}{|c|c|c|c|c|c|}
\hline & Lag & df & RR & $95 \% \mathrm{Cl}$ for $\mathrm{RR}$ & $P$-Value \\
\hline \multirow[t]{6}{*}{ Lag 0} & $\mathrm{O}_{3}(\mathrm{ppb})$ & 7.19 & 1.03 & $1.00-1.07$ & 0.037 \\
\hline & $\mathrm{CO}(\mathrm{ppm})$ & 4.88 & 0.98 & $0.94-1.01$ & 0.235 \\
\hline & $\mathrm{NO}_{2}(\mathrm{ppb})$ & 2.33 & 1.03 & $1.00-1.06$ & 0.045 \\
\hline & $\mathrm{SO}_{2}(\mathrm{ppb})$ & 1 & 0.96 & $0.92-1.01$ & 0.093 \\
\hline & $\mathrm{PM}_{10}\left(\mu \mathrm{g} / \mathrm{m}^{3}\right)$ & 7.50 & 1.03 & $1.02-1.05$ & $<0.001$ \\
\hline & $\mathrm{PM}_{2.5}\left(\mu \mathrm{g} / \mathrm{m}^{3}\right)$ & 3.92 & 1.02 & $0.97-1.08$ & 0.156 \\
\hline \multirow[t]{6}{*}{ Lag 1} & $\mathrm{O}_{3}(\mathrm{ppb})$ & 3.20 & 1.03 & $0.99-1.06$ & 0.100 \\
\hline & $\mathrm{CO}(\mathrm{ppm})$ & 6.45 & 0.97 & $0.94-1.01$ & 0.107 \\
\hline & $\mathrm{NO}_{2}(\mathrm{ppb})$ & 4.47 & 1.04 & $1.00-1.06$ & 0.019 \\
\hline & $\mathrm{SO}_{2}(\mathrm{ppb})$ & 3.15 & 0.95 & $0.91-0.99$ & 0.032 \\
\hline & $\mathrm{PM}_{10}\left(\mu \mathrm{g} / \mathrm{m}^{3}\right)$ & 6.69 & 1.02 & $1.01-1.04$ & $<0.001$ \\
\hline & $\mathrm{PM}_{2.5}\left(\mu \mathrm{g} / \mathrm{m}^{3}\right)$ & 4.95 & 1.04 & $1.02-1.07$ & 0.003 \\
\hline \multirow[t]{6}{*}{$\operatorname{Lag} 2$} & $\mathrm{O}_{3}(\mathrm{ppb})$ & 3.31 & 1.00 & $0.97-1.04$ & 0.394 \\
\hline & $\mathrm{CO}(\mathrm{ppm})$ & 4.85 & 0.96 & $0.93-1.00$ & 0.073 \\
\hline & $\mathrm{NO}_{2}(\mathrm{ppb})$ & 4.15 & 1.03 & $1.00-1.07$ & 0.028 \\
\hline & $\mathrm{SO}_{2}(\mathrm{ppb})$ & 1.30 & 0.95 & $0.91-0.99$ & 0.030 \\
\hline & $\mathrm{PM}_{10}\left(\mu \mathrm{g} / \mathrm{m}^{3}\right)$ & 6.49 & 1.02 & $1.01-1.03$ & 0.002 \\
\hline & $\mathrm{PM}_{2.5}\left(\mu \mathrm{g} / \mathrm{m}^{3}\right)$ & 2.09 & 1.04 & $1.02-1.05$ & 0.014 \\
\hline \multirow[t]{6}{*}{ Lag 3} & $\mathrm{O}_{3}(\mathrm{ppb})$ & 3.92 & 1.03 & $0.99-1.06$ & 0.053 \\
\hline & CO (ppm) & 5.60 & 1.07 & 0.99-1.08 & 0.488 \\
\hline & $\mathrm{NO}_{2}(\mathrm{ppb})$ & 2.79 & 1.02 & 0.99-1.06 & 0.125 \\
\hline & $\mathrm{SO}_{2}(\mathrm{ppb})$ & 8.21 & 0.94 & $0.91-0.99$ & 0.016 \\
\hline & $\mathrm{PM}_{10}\left(\mu \mathrm{g} / \mathrm{m}^{3}\right)$ & 6.43 & 1.02 & $1.01-1.03$ & 0.004 \\
\hline & $\mathrm{PM}_{2.5}\left(\mu \mathrm{g} / \mathrm{m}^{3}\right)$ & 2.41 & 1.05 & $0.99-1.10$ & 0.051 \\
\hline \multirow{6}{*}{$\begin{array}{l}\text { Mean } \\
\text { Lag 4-8 }\end{array}$} & $\mathrm{O}_{3}(\mathrm{ppb})$ & 3.33 & 1.03 & $0.99-1.06$ & 0.066 \\
\hline & $\mathrm{CO}(\mathrm{ppm})$ & 5.52 & 1.03 & $0.99-1.07$ & 0.311 \\
\hline & $\mathrm{NO}_{2}(\mathrm{ppb})$ & 2.54 & 1.02 & 0.99-1.06 & 0.116 \\
\hline & $\mathrm{SO}_{2}(\mathrm{ppb})$ & 2.82 & 0.97 & $0.92-1.01$ & 0.117 \\
\hline & $\mathrm{PM}_{10}\left(\mathrm{\mu g} / \mathrm{m}^{3}\right)$ & 6.47 & 1.00 & $0.99-1.01$ & 0.089 \\
\hline & $\mathrm{PM}_{2.5}\left(\mu \mathrm{g} / \mathrm{m}^{3}\right)$ & 3.84 & 0.93 & $0.89-0.98$ & 0.011 \\
\hline
\end{tabular}

safely omitted from the analysis. Also in this study, we assessed the short-term effect of exposure; and population change cannot have a significant effect on shortterm results [17].

In this study, maximum daily concentration of ozone $\left(\mathrm{O}_{3}\right)$ was $77.09 \mathrm{ppb}$ and lower than WHO guidelines (Table 2). $\mathrm{O}_{3}$ also was related to overall respiratory deaths, male respiratory deaths and respiratory deaths in people 18 to 60 years and in people over 60 years old. $\mathrm{RR}$ for overall respiratory deaths was 1.03 (95\% CI: 1.01-1.05) per $10 \mathrm{ppb}$ increase in $\mathrm{O}_{3}$ in lag 1. This relation between $\mathrm{O}_{3}$ and respiratory deaths has also been seen in other studies. Monthly concentration of $\mathrm{O}_{3}$ was related to respiratory death in people over 60 years old 
Table 5 Results of adjusted Generalized Additive Model and the effect of pollutants on respiratory deaths in women (for 1 unit increase in $\mathrm{CO}$ and 10 units increase in all other pollutants)

\begin{tabular}{|c|c|c|c|c|c|}
\hline & Lag & df & RR & $95 \% \mathrm{Cl}$ for $\mathrm{RR}$ & $P$-Value \\
\hline \multirow[t]{6}{*}{ Lag 0} & $\mathrm{O}_{3}(\mathrm{ppb})$ & 7.89 & 1.01 & $0.97-1.05$ & 0.616 \\
\hline & $\mathrm{CO}(\mathrm{ppm})$ & 4.62 & 0.98 & $0.94-1.03$ & 0.438 \\
\hline & $\mathrm{NO}_{2}(\mathrm{ppb})$ & 3.80 & 1.06 & $1.03-1.10$ & $<0.001$ \\
\hline & $\mathrm{SO}_{2}(\mathrm{ppb})$ & 5.27 & 0.94 & $0.89-0.99$ & 0.019 \\
\hline & $\mathrm{PM}_{10}\left(\mu \mathrm{g} / \mathrm{m}^{3}\right)$ & 4.53 & 1.03 & $1.01-1.04$ & $<0.001$ \\
\hline & $\mathrm{PM}_{2.5}\left(\mu \mathrm{g} / \mathrm{m}^{3}\right)$ & 1.83 & 1.04 & $0.98-1.10$ & 0.355 \\
\hline \multirow[t]{6}{*}{ Lag 1} & $\mathrm{O}_{3}(\mathrm{ppb})$ & 4.49 & 1.02 & $0.98-1.06$ & 0.270 \\
\hline & $\mathrm{CO}(\mathrm{ppm})$ & 5.39 & 1.03 & $0.99-1.08$ & 0.476 \\
\hline & $\mathrm{NO}_{2}(\mathrm{ppb})$ & 6.29 & 1.05 & $1.04-1.09$ & 0.005 \\
\hline & $\mathrm{SO}_{2}(\mathrm{ppb})$ & 4.33 & 0.95 & $0.91-1.00$ & 0.056 \\
\hline & $\mathrm{PM}_{10}\left(\mu \mathrm{g} / \mathrm{m}^{3}\right)$ & 5.72 & 1.01 & $0.99-1.03$ & 0.063 \\
\hline & $\mathrm{PM}_{2.5}\left(\mu \mathrm{g} / \mathrm{m}^{3}\right)$ & 7.23 & 1.05 & $1.01-1.09$ & $<0.001$ \\
\hline \multirow[t]{6}{*}{ Lag 2} & $\mathrm{O}_{3}(\mathrm{ppb})$ & 7.19 & 1.03 & $0.99-1.07$ & 0.178 \\
\hline & $\mathrm{CO}(\mathrm{ppm})$ & 5.76 & 1.04 & 0.99-1.09 & 0.694 \\
\hline & $\mathrm{NO}_{2}(\mathrm{ppb})$ & 3.63 & 1.04 & $1.01-1.08$ & 0.010 \\
\hline & $\mathrm{SO}_{2}(\mathrm{ppb})$ & 5.27 & 0.94 & 0.89-0.99 & 0.029 \\
\hline & $\mathrm{PM}_{10}\left(\mu \mathrm{g} / \mathrm{m}^{3}\right)$ & 6.61 & 1.01 & $0.99-1.03$ & 0.113 \\
\hline & $\mathrm{PM}_{2.5}\left(\mu \mathrm{g} / \mathrm{m}^{3}\right)$ & 2.53 & 1.03 & $1.01-1.05$ & 0.003 \\
\hline \multirow[t]{6}{*}{ Lag 3} & $\mathrm{O}_{3}(\mathrm{ppb})$ & 6.45 & 1.03 & $0.98-1.06$ & 0.174 \\
\hline & $\mathrm{CO}(\mathrm{ppm})$ & 4.91 & 1.04 & $0.99-1.09$ & 0.421 \\
\hline & $\mathrm{NO}_{2}(\mathrm{ppb})$ & 2.22 & 1.05 & $1.02-1.09$ & 0.003 \\
\hline & $\mathrm{SO}_{2}(\mathrm{ppb})$ & 1.36 & 0.96 & $0.92-1.01$ & 0.132 \\
\hline & $\mathrm{PM}_{10}\left(\mu \mathrm{g} / \mathrm{m}^{3}\right)$ & 5.96 & 1.01 & $0.99-1.03$ & 0.071 \\
\hline & $\mathrm{PM}_{2.5}\left(\mu \mathrm{g} / \mathrm{m}^{3}\right)$ & 2.41 & 1.03 & $0.98-1.07$ & 0.067 \\
\hline \multirow{6}{*}{$\begin{array}{l}\text { Mean } \\
\text { Lag 4-8 }\end{array}$} & $\mathrm{O}_{3}(\mathrm{ppb})$ & 3.31 & 1.01 & $0.96-1.04$ & 0.801 \\
\hline & $\mathrm{CO}(\mathrm{ppm})$ & 5.29 & 0.98 & $0.96-1.01$ & 0.124 \\
\hline & $\mathrm{NO}_{2}(\mathrm{ppb})$ & 4.88 & 1.05 & $1.01-1.08$ & 0.011 \\
\hline & $\mathrm{SO}_{2}(\mathrm{ppb})$ & 4.61 & 0.95 & $0.90-1.00$ & 0.052 \\
\hline & $\mathrm{PM}_{10}\left(\mu \mathrm{g} / \mathrm{m}^{3}\right)$ & 6.93 & 1.00 & $0.98-1.02$ & 0.589 \\
\hline & $\mathrm{PM}_{2.5}\left(\mu \mathrm{g} / \mathrm{m}^{3}\right)$ & 2.68 & 0.95 & $0.90-1.01$ & 0.131 \\
\hline
\end{tabular}

in Shiraz (South West Iran) and was RR $=1.04,95 \%$ CI: 1.00-1.09 per one ppb increase in $\mathrm{O}_{3}$ [9], and in Kerman (south east of Iran), there was a significant relation between the monthly concentration of $\mathrm{O}_{3}$ and respiratory deaths in men and was $\mathrm{RR}=1.03,95 \% \mathrm{CI}$ : 1.01-1.04 per one ppm increase in $\mathrm{O}_{3}$ [16]. Shahi et al. showed that respiratory admissions to hospitals were related to $\mathrm{O}_{3}$ in lag 0 to 7 days in Tehran, the highest RR was seen in lag 1.01 (95\% CI: 1.01-1.02) [18]. In this study, the mean concentration of $\mathrm{O}_{3}$ was $24.66 \pm 9.12$ and more than Shiraz $(17.49 \pm 16.28 \mathrm{ppb})$ [9] and Kerman $(0.02 \pm 0.01 \mathrm{ppm})$ [16]. In the period of this study, on only 6 days the concentration of $\mathrm{SO}_{2}$ passed WHO thresholds.
Table 6 Results of adjusted Generalized Additive Model, about the effect of air pollutants on respiratory deaths in peoples under 18 years old (for 1 unit increase in $\mathrm{CO}$ and 10 units increase in all other pollutants)

\begin{tabular}{|c|c|c|c|c|c|}
\hline & Lag & df & $R R$ & $95 \% \mathrm{Cl}$ for RR & $P$-Value \\
\hline \multirow[t]{6}{*}{ Lag 0} & $\mathrm{O}_{3}(\mathrm{ppb})$ & 2.01 & 0.93 & $0.86-1.01$ & 0.073 \\
\hline & CO (ppm) & 1.54 & 0.94 & $0.86-1.02$ & 0.134 \\
\hline & $\mathrm{NO}_{2}(\mathrm{ppb})$ & 1 & 1.01 & $0.97-1.08$ & 0.826 \\
\hline & $\mathrm{SO}_{2}(\mathrm{ppb})$ & 3.24 & 0.97 & $0.86-1.05$ & 0.286 \\
\hline & $\mathrm{PM}_{10}\left(\mu \mathrm{g} / \mathrm{m}^{3}\right)$ & 3.48 & 1.01 & $0.98-1.05$ & 0.353 \\
\hline & $\mathrm{PM}_{2.5}\left(\mu \mathrm{g} / \mathrm{m}^{3}\right)$ & 6.71 & 0.99 & $0.88-1.16$ & 0.981 \\
\hline \multirow[t]{6}{*}{ Lag 1} & $\mathrm{O}_{3}(\mathrm{ppb})$ & 2.61 & 0.95 & $0.87-1.02$ & 0.153 \\
\hline & $\mathrm{CO}$ (ppm) & 1.79 & 0.92 & $0.84-1.00$ & 0.060 \\
\hline & $\mathrm{NO}_{2}(\mathrm{ppb})$ & 3.79 & 0.98 & $0.91-1.06$ & 0.647 \\
\hline & $\mathrm{SO}_{2}(\mathrm{ppb})$ & 4.71 & 0.95 & $0.85-1.05$ & 0.281 \\
\hline & $\mathrm{PM}_{10}\left(\mu \mathrm{g} / \mathrm{m}^{3}\right)$ & 5.02 & 0.99 & $0.96-1.03$ & 0.864 \\
\hline & $\mathrm{PM}_{2.5}\left(\mu \mathrm{g} / \mathrm{m}^{3}\right)$ & 2.46 & 1.00 & $0.88-1.13$ & 0.95 \\
\hline \multirow[t]{6}{*}{ Lag 2} & $\mathrm{O}_{3}(\mathrm{ppb})$ & 4.52 & 0.95 & $0.88-1.03$ & 0.246 \\
\hline & $\mathrm{CO}$ (ppm) & 3.84 & 0.89 & $0.82-0.98$ & 0.017 \\
\hline & $\mathrm{NO}_{2}(\mathrm{ppb})$ & 1 & 0.98 & $0.91-1.05$ & 0.559 \\
\hline & $\mathrm{SO}_{2}(\mathrm{ppb})$ & 4.5 & 1.01 & $0.91-1.11$ & 0.918 \\
\hline & $\mathrm{PM}_{10}\left(\mu \mathrm{g} / \mathrm{m}^{3}\right)$ & 5.03 & 0.99 & $0.97-1.03$ & 0.894 \\
\hline & $\mathrm{PM}_{2.5}\left(\mu \mathrm{g} / \mathrm{m}^{3}\right)$ & 1 & 1.02 & $0.91-1.15$ & 0.688 \\
\hline \multirow[t]{6}{*}{ Lag 3} & $\mathrm{O}_{3}(\mathrm{ppb})$ & 2.38 & 0.94 & $0.87-1.01$ & 0.107 \\
\hline & $\mathrm{CO}$ (ppm) & 1 & 0.87 & $0.80-0.95$ & 0.002 \\
\hline & $\mathrm{NO}_{2}(\mathrm{ppb})$ & 5.41 & 0.99 & $0.93-1.07$ & 0.901 \\
\hline & $\mathrm{SO}_{2}(\mathrm{ppb})$ & 8.25 & 0.99 & 0.89-1.09 & 0.899 \\
\hline & $\mathrm{PM}_{10}\left(\mathrm{\mu g} / \mathrm{m}^{3}\right)$ & 2.75 & 1.00 & $0.97-1.03$ & 0.973 \\
\hline & $\mathrm{PM}_{2.5}\left(\mu \mathrm{g} / \mathrm{m}^{3}\right)$ & 6.15 & 1.03 & $0.91-1.16$ & 0.647 \\
\hline \multirow{6}{*}{$\begin{array}{l}\text { Mean } \\
\text { Lag 4-8 }\end{array}$} & $\mathrm{O}_{3}(\mathrm{ppb})$ & 3.28 & 0.93 & $0.86-1.01$ & 0.078 \\
\hline & CO (ppm) & 1 & 0.91 & $0.83-0.99$ & 0.034 \\
\hline & $\mathrm{NO}_{2}(\mathrm{ppb})$ & 1.33 & 1.04 & $0.96-1.11$ & 0.344 \\
\hline & $\mathrm{SO}_{2}(\mathrm{ppb})$ & 5.14 & 0.94 & $0.85-1.07$ & 0.272 \\
\hline & $\mathrm{PM}_{10}\left(\mu \mathrm{g} / \mathrm{m}^{3}\right)$ & 2.7 & 1.00 & $0.97-1.03$ & 0.961 \\
\hline & $\mathrm{PM}_{2.5}\left(\mu \mathrm{g} / \mathrm{m}^{3}\right)$ & 1 & 1.04 & $0.92-1.17$ & 0.523 \\
\hline
\end{tabular}

In this study, $\mathrm{NO}_{2}$ had a significant relationship with respiratory mortality in both sexes and in groups aged 18 and over. Other studies have also seen a relation between nitrogen dioxide and respiratory mortality [19-21]. The strongest $\mathrm{RR}$ for $\mathrm{NO}_{2}$ in this study for overall death was 1.04 (95\% CI; $1.02-1.04)$ in lag 1. In César et al.'s study in Brazil, the RR for respiratory deaths per $10 \mu \mathrm{g} / \mathrm{m}^{3}$ increase in NOx was 1.04 (95\% CI: 1.01-1.06) [19]. In Zhang et al. in Beijing, China the RR for respiratory death was 1.01 (95\% CI: 1.00 1.01) per $10 \mu \mathrm{g} / \mathrm{m} 3$ increase of $\mathrm{NO}_{2}$ in lag $0-2$ days [21]. In a meta-analysis in China, the pooled estimate of RR for respiratory deaths was 1.01 (95\% CI: 
Table 7 Results of adjusted Generalized Additive Model and the effect of pollutants on respiratory deaths in peoples 18-60 years old (for 1 unit increase in $\mathrm{CO}$ and 10 units increase in all other pollutants)

\begin{tabular}{|c|c|c|c|c|c|}
\hline & Lag & $d f$ & RR & $95 \% \mathrm{Cl}$ for RR & $P$ - Value \\
\hline \multirow[t]{6}{*}{ Lag 0} & $\mathrm{O}_{3}(\mathrm{ppb})$ & 7.57 & 1.07 & 0.99-1.09 & 0.128 \\
\hline & $\mathrm{CO}(\mathrm{ppm})$ & 6.33 & 0.99 & $0.93-1.04$ & 0.549 \\
\hline & $\mathrm{NO}_{2}(\mathrm{ppb})$ & 1 & 1.05 & $1.00-1.09$ & 0.034 \\
\hline & $\mathrm{SO}_{2}(\mathrm{ppb})$ & 3.19 & 0.96 & $0.90-1.02$ & 0.195 \\
\hline & $\mathrm{PM}_{10}\left(\mu \mathrm{g} / \mathrm{m}^{3}\right)$ & 7.47 & 1.02 & $1.00-1.04$ & $<0.001$ \\
\hline & $\mathrm{PM}_{2.5}\left(\mu \mathrm{g} / \mathrm{m}^{3}\right)$ & 3.25 & 1.05 & $0.96-1.13$ & 0.198 \\
\hline \multirow[t]{6}{*}{ Lag 1} & $\mathrm{O}_{3}(\mathrm{ppb})$ & 5.57 & 1.04 & $0.99-1.08$ & 0.117 \\
\hline & $\mathrm{CO}$ (ppm) & 5.14 & 0.97 & $0.93-1.03$ & 0.345 \\
\hline & $\mathrm{NO}_{2}(\mathrm{ppb})$ & 7.07 & 1.05 & $1.01-1.10$ & 0.026 \\
\hline & $\mathrm{SO}_{2}(\mathrm{ppb})$ & 1 & 0.96 & $0.90-1.02$ & 0.206 \\
\hline & $\mathrm{PM}_{10}\left(\mu \mathrm{g} / \mathrm{m}^{3}\right)$ & 7.08 & 1.03 & $1.01-1.05$ & 0.001 \\
\hline & $\mathrm{PM}_{2.5}\left(\mu \mathrm{g} / \mathrm{m}^{3}\right)$ & 2.87 & 1.06 & $1.02-1.06$ & $<0.001$ \\
\hline \multirow[t]{6}{*}{$\operatorname{Lag} 2$} & $\mathrm{O}_{3}(\mathrm{ppb})$ & 5.28 & 1.06 & $1.01-1.11$ & 0.014 \\
\hline & $\mathrm{CO}(\mathrm{ppm})$ & 4.98 & 0.98 & $0.96-1.04$ & 0.597 \\
\hline & $\mathrm{NO}_{2}(\mathrm{ppb})$ & 4.14 & 1.06 & $1.01-1.10$ & 0.011 \\
\hline & $\mathrm{SO}_{2}(\mathrm{ppb})$ & 1.61 & 0.95 & $0.88-1.01$ & 0.087 \\
\hline & $\mathrm{PM}_{10}\left(\mu \mathrm{g} / \mathrm{m}^{3}\right)$ & 6.84 & 1.03 & $1.01-1.05$ & 0.003 \\
\hline & $\mathrm{PM}_{2.5}\left(\mu \mathrm{g} / \mathrm{m}^{3}\right)$ & 3.16 & 1.04 & $1.01-1.07$ & $<0.001$ \\
\hline \multirow[t]{6}{*}{ Lag 3} & $\mathrm{O}_{3}(\mathrm{ppb})$ & 6.96 & 1.07 & $1.02-1.12$ & 0.004 \\
\hline & $\mathrm{CO}$ (ppm) & 6.99 & 0.97 & $0.92-1.02$ & 0.250 \\
\hline & $\mathrm{NO}_{2}(\mathrm{ppb})$ & 2.2 & 1.04 & $0.99-1.10$ & 0.084 \\
\hline & $\mathrm{SO}_{2}(\mathrm{ppb})$ & 8.61 & 0.95 & 0.89-1.01 & 0.120 \\
\hline & $\mathrm{PM}_{10}\left(\mu \mathrm{g} / \mathrm{m}^{3}\right)$ & 7.47 & 1.02 & 0.95-1.09 & 0.156 \\
\hline & $\mathrm{PM}_{2.5}\left(\mu \mathrm{g} / \mathrm{m}^{3}\right)$ & 3.21 & 1.02 & $0.95-1.09$ & 0.094 \\
\hline \multirow{6}{*}{$\begin{array}{l}\text { Mean } \\
\text { Lag 4-8 }\end{array}$} & $\mathrm{O}_{3}(\mathrm{ppb})$ & 8.61 & 1.04 & 0.99-1.09 & 0.119 \\
\hline & CO (ppm) & 4.49 & 0.97 & $0.93-1.03$ & 0.433 \\
\hline & $\mathrm{NO}_{2}(\mathrm{ppb})$ & 2.7 & 1.03 & $0.98-1.07$ & 0.243 \\
\hline & $\mathrm{SO}_{2}(\mathrm{ppb})$ & 1 & 0.95 & $0.89-1.02$ & 0.137 \\
\hline & $\mathrm{PM}_{10}\left(\mu \mathrm{g} / \mathrm{m}^{3}\right)$ & 7.73 & 1.02 & $1.00-1.04$ & 0.017 \\
\hline & $\mathrm{PM}_{2.5}\left(\mu \mathrm{g} / \mathrm{m}^{3}\right)$ & 4.26 & 1.00 & $0.97-1.04$ & 0.341 \\
\hline
\end{tabular}

1.01-1.02) per $10 \mu \mathrm{g} / \mathrm{m}^{3}$ increase in $\mathrm{NO}_{2}$ [22], which is close to our results.

In Tehran, the major source of $\mathrm{NO}_{2}$ production is the transportation system. Therefore, it is necessary to increase the use of public transport and decrease the use of fossil fuels to reduce this ambient air pollutant.

In this study, particulate matters $\left(\mathrm{PM}_{10}\right.$ and $\left.\mathrm{PM}_{2.5}\right)$ showed a direct relation with respiratory deaths which were $\mathrm{RR}=1.03,95 \% \mathrm{CI}: 1.02-1.04$ and $\mathrm{RR}=1.05$, 95\% CI: 1.01-1.09 respectively. Similar to this study, in studies done by Yang et al., in Beijing, China, there was a 1.7\% (95\% CI: 0.01-3.32) increase of daily respiratory deaths associated with an interquartile range (IQR)
Table 8 Results of adjusted Generalized Additive Model and the effect of pollutants on respiratory deaths in peoples over 60 years old (for 1 unit increase in $\mathrm{CO}$ and 10 units increase in all other pollutants)

\begin{tabular}{|c|c|c|c|c|c|}
\hline & Lag & $d f$ & RR & $95 \% \mathrm{Cl}$ for $\mathrm{RR}$ & $P$-Value \\
\hline \multirow[t]{6}{*}{ Lag 0} & $\mathrm{O}_{3}(\mathrm{ppb})$ & 8.18 & 1.04 & $1.00-1.07$ & 0.024 \\
\hline & $\mathrm{CO}$ (ppm) & 4.39 & 0.98 & $0.95-1.02$ & 0.312 \\
\hline & $\mathrm{NO}_{2}(\mathrm{ppb})$ & 3.38 & 1.05 & $1.02-1.08$ & 0.001 \\
\hline & $\mathrm{SO}_{2}(\mathrm{ppb})$ & 2.58 & 0.96 & $0.92-0.99$ & 0.033 \\
\hline & $\mathrm{PM}_{10}\left(\mu \mathrm{g} / \mathrm{m}^{3}\right)$ & 7.29 & 1.03 & $1.02-1.04$ & $<0.001$ \\
\hline & $\mathrm{PM}_{2.5}\left(\mu \mathrm{g} / \mathrm{m}^{3}\right)$ & 2.64 & 1.03 & $1.01-1.06$ & 0.039 \\
\hline \multirow[t]{6}{*}{ Lag 1} & $\mathrm{O}_{3}(\mathrm{ppb})$ & 3.48 & 1.04 & $1.00-1.07$ & 0.027 \\
\hline & $\mathrm{CO}(\mathrm{ppm})$ & 6.44 & 0.97 & $0.93-1.00$ & 0.054 \\
\hline & $\mathrm{NO}_{2}(\mathrm{ppb})$ & 4.67 & 1.04 & $1.01-1.07$ & 0.01 \\
\hline & $\mathrm{SO}_{2}(\mathrm{ppb})$ & 2.91 & 0.95 & $0.91-0.99$ & 0.023 \\
\hline & $\mathrm{PM}_{10}\left(\mu \mathrm{g} / \mathrm{m}^{3}\right)$ & 5.77 & 1.02 & $1.01-1.03$ & 0.005 \\
\hline & $\mathrm{PM}_{2.5}\left(\mu \mathrm{g} / \mathrm{m}^{3}\right)$ & 2.55 & 1.04 & $1.02-1.08$ & 0.002 \\
\hline \multirow[t]{6}{*}{$\operatorname{Lag} 2$} & $\mathrm{O}_{3}(\mathrm{ppb})$ & 4.02 & 1.03 & $1.00-1.06$ & 0.041 \\
\hline & $\mathrm{CO}$ (ppm) & 7 & 1.01 & $0.96-1.07$ & 0.232 \\
\hline & $\mathrm{NO}_{2}(\mathrm{ppb})$ & 3.86 & 1.04 & $1.01-1.07$ & 0.006 \\
\hline & $\mathrm{SO}_{2}(\mathrm{ppb})$ & 3.57 & 0.95 & $0.91-0.98$ & 0.009 \\
\hline & $\mathrm{PM}_{10}\left(\mu \mathrm{g} / \mathrm{m}^{3}\right)$ & 6.24 & 1.01 & $1.00-1.03$ & 0.038 \\
\hline & $\mathrm{PM}_{2.5}\left(\mu \mathrm{g} / \mathrm{m}^{3}\right)$ & 1.7 & 1.03 & $1.01-1.05$ & 0.007 \\
\hline \multirow[t]{6}{*}{ Lag 3} & $\mathrm{O}_{3}(\mathrm{ppb})$ & 4.78 & 1.03 & $0.99-1.06$ & 0.065 \\
\hline & $\mathrm{CO}$ (ppm) & 5.09 & 1.03 & $0.99-1.07$ & 0.212 \\
\hline & $\mathrm{NO}_{2}(\mathrm{ppb})$ & 2.89 & 1.04 & $1.01-1.07$ & 0.003 \\
\hline & $\mathrm{SO}_{2}(\mathrm{ppb})$ & 1.34 & 0.95 & $0.91-0.98$ & 0.01 \\
\hline & $\mathrm{PM}_{10}\left(\mu \mathrm{g} / \mathrm{m}^{3}\right)$ & 5.81 & 1.01 & $1.00-1.03$ & 0.026 \\
\hline & $\mathrm{PM}_{2.5}\left(\mu \mathrm{g} / \mathrm{m}^{3}\right)$ & 2.11 & 1.02 & $0.98-1.04$ & 0.265 \\
\hline \multirow{6}{*}{$\begin{array}{l}\text { Mean } \\
\text { Lag 4-8 }\end{array}$} & $\mathrm{O}_{3}(\mathrm{ppb})$ & 3.18 & 1.03 & $0.99-1.06$ & 0.078 \\
\hline & CO (ppm) & 5.05 & 1.03 & $0.99-1.07$ & 0.241 \\
\hline & $\mathrm{NO}_{2}(\mathrm{ppb})$ & 4.98 & 1.05 & $1.02-1.08$ & 0.006 \\
\hline & $\mathrm{SO}_{2}(\mathrm{ppb})$ & 7.87 & 0.92 & $0.89-0.96$ & 0.001 \\
\hline & $\mathrm{PM}_{10}\left(\mu \mathrm{g} / \mathrm{m}^{3}\right)$ & 7.83 & 1.01 & $1.00-1.03$ & 0.035 \\
\hline & $\mathrm{PM}_{2.5}\left(\mu \mathrm{g} / \mathrm{m}^{3}\right)$ & 1.35 & 0.96 & $0.92-1.01$ & 0.11 \\
\hline
\end{tabular}

increase in $\mathrm{PM}_{10}$ concentrations [23] and in a study by Wang et al., in Seoul, Korea the RR for respiratory death was 1.0001 (95\% CI: $1.0001-1.0002)$ per $1 \mu \mathrm{g} / \mathrm{m}^{3}$ increase in $\mathrm{PM}_{10}$ in lag 0 and 1 [14]. Another study by Lopez et al., in the Canary Islands showed that the Rate Ratio for respiratory mortality was $\mathrm{RR}=1.04$ (95\% CI: $1.01-1.08)$ by $10 \mu \mathrm{g} / \mathrm{m}^{3}$ increase in $\mathrm{PM}_{10}$ and the maximum effect of exposure to $\mathrm{PM}_{10}$ on respiratory death was on the same day [4]. Studies from England [24], and Kerman, Iran [16] also showed a significant relation between monthly concentrations of $\mathrm{PM}_{10}$ and respiratory deaths. In a study from Isfahan, Iran there was a significant relation between $\mathrm{PM}_{10}$ and the number of children 
admitted to hospitals due to respiratory problems [25]. In Wang et al.'s, study in Seoul, Korea $\left(71.23 \mu \mathrm{g} / \mathrm{m}^{3}\right)$ [14], Yang et al., in Beijing, China $\left(96.3 \mu \mathrm{g} / \mathrm{m}^{3}\right)$ [23], Khanjani et al, in Kerman, Iran $\left(125.69 \mu \mathrm{g} / \mathrm{m}^{3}\right)$ [16] and Mansourian et al, in Isfahan, Iran $\left(118.04 \mu \mathrm{g} / \mathrm{m}^{3}\right)$ [25] the mean of concentration of $\mathrm{PM}_{10}$ were higher than this study $\left(69.75 \mu \mathrm{g} / \mathrm{m}^{3}\right)$, but in Lopez et al, in the Canary Islands $\left(37.7 \mu \mathrm{g} / \mathrm{m}^{3}\right)$ [4] and a meta-analysis by Hansel et al, in England $\left(20.7 \mu \mathrm{g} / \mathrm{m}^{3}\right)$ [24] the mean concentration of $\mathrm{PM}_{10}$ was lower than this study. Particulate matter are one of the most important pollutants that affect human health. Particulate matter compounds and sizes vary in different places and this can explain different study results.

In this study, Carbon monoxide showed no significant relation, and $\mathrm{SO}_{2}$ showed inverse relations with respiratory death, that was likely caused by the harvesting effect.

In some studies $\mathrm{CO}$ and $\mathrm{SO}_{2}$ have shown relations with respiratory deaths. For example, monthly $\mathrm{CO}$ concentrations were related with respiratory deaths in Shiraz [9] and CO was also related with respiratory hospital admission in Tehran [18].

$\mathrm{SO}_{2}$ in the Canary Islands [4], Kerman, Iran [16], Taiwan [26], and Tehran [20], was reported to be related with respiratory death. Other studies from Kerman reported a relation between hospital respiratory admission and $\mathrm{SO}_{2}$ [27].

The effects of different ambient air pollutants, has been reported differently in different parts of the world. This variability may be related to different study designs, various statistical models and including or not including different confounders.

In this study and other similar studies $[4,9,16,25,26]$, some air pollutants had more adverse effects on the health outcomes in men than in women. In this study only ozone showed a different result based on gender and was more effective on respiratory deaths in men. The reason might be that men spend more time in outdoor environments. One study conducted in Tehran showed that the concentrations of ambient (outdoor) $\mathrm{PM}_{10}, \mathrm{PM}_{2.5}$ and $\mathrm{PM}_{1}$ were higher than indoors [28].

In this study, older people were more vulnerable to exposure to pollutants than younger people. None of the air pollutants were related to respiratory death in people under 18 years old. But in the over 18 year age groups; $\mathrm{NO}_{2}, \mathrm{O}_{3}, \mathrm{PM}_{10}$ and $\mathrm{PM}_{2.5}$ showed relations with respiratory death.

Mobile and stationary sources are both responsible for air pollution in Tehran. Motor vehicles are the main source for especially $\mathrm{CO}, \mathrm{NO}_{2}$ and $\mathrm{PM}_{10}$ in Tehran. Tehran also has a lot of factories and is an industrial hub. According to Mazaheri et al., mobile sources of air pollution are more important than stationary sources in making and emitting $\mathrm{NO}_{2}$ and $\mathrm{CO}$ in Tehran [29]. Wang et al [30] showed that the mass ratio of nitrate to sulfate is a good indicator of the relative importance of mobile to stationary sources of nitrogen and sulfur in the atmosphere and it is reasonable to use nitrate as an indicator of transportation emission and sulfate as an indicator of stationary emission. The nitrate/sulfate ratio in Tehran, in 2014 ranged from 1.17 to 1.21 for $\mathrm{PM}_{10}, 1.16$ to 1.40 for $\mathrm{PM}_{2.5}$, and 1.23 to 1.45 for $\mathrm{PM}_{1}$. The results indicated that mobile sources were more important source of PM in Tehran [28]. The dismissal of old cars, using less fossil fuels for transportation and heating; and the transfer of factories out of the city are recommended in order to decrease air pollution in Tehran.

Another source of air pollution are the Middle Eastern dust storms which have also affected western and central Iran (including Tehran). Regional and international efforts to reduce particle matter in the Middle East are necessary.

Other studies about the effects of air pollutants indicate that air pollution has also caused deaths and hospital admissions due to heart disease and road accidents in Iran [31-36]. This evidence further shows the importance of controlling air pollution in this country.

Some limitations of this study were that the impact of other variables such as the concentrations of other pollutants like NO, lead and total hydrocarbons (THC), or wind direction was not investigated. We did not have data about influenza epidemics in Tehran and we did not adjust for this variable. Finally, given the ecological nature of this study, results cannot be easily inferred to an individual level.

\section{Conclusions}

The results indicate that ambient Particle matter, Ozone and Nitrogen dioxide can be responsible for a significant part of respiratory mortalities in Tehran. Additional efforts to control and reduce air pollutant is necessary.

\section{Acknowledgements \\ We appreciate the Tehran Air Quality Control Company (AQCC), Tehran Department of Environmental Protection, Tehran Meteorological Organization and the Tehran Cemetery (Behesht-e-Zahra) for providing the data.}

\section{Funding}

This study was supported by Grant No. 95-267, from Kerman University of Medical Sciences, Kerman, Iran.

\section{Availability of data and materials}

We cannot share data at this stage, because the owner of the air pollution data is the Tehran Environmental Protection Agency and the death registry of Tehran. We need to consult with them before disclosing data.

\section{Authors' contributions}

NK initiated the idea for the study and was the main supervisor, helped in writing and editing the manuscript. AD acquired the data, cleaned the data, analyzed the data and prepared the initial draft. AB supervised data analysis, provided statistical consultation and edited the final manuscript. GG provided scientific advice for air pollution and edited the final article. MY provided scientific and methodology consultation, edited the final manuscript. All authors read and approved the final manuscript. 


\section{Ethics approval and consent to participate}

This project was approved by the Standing Committee of Ethics in Research of Kerman University of Medical Sciences (Ethics code: IR.KMU.REC.1394.267).

\section{Consent for publication}

Not applicable.

\section{Competing interests}

The authors declare that they have no competing interests.

\section{Publisher's Note}

Springer Nature remains neutral with regard to jurisdictional claims in published maps and institutional affiliations.

\section{Author details}

${ }^{1}$ Neurology Research Center, Kerman University of Medical Sciences, Kerman, Iran. ${ }^{2}$ Environmental Health Engineering Research Center, Kerman University of Medical Sciences, Kerman, Iran. ${ }^{3}$ Monash Centre for Occupational \& Environmental Health, School of Public Health and Preventive Medicine, Monash University, Melbourne, Australia. ${ }^{4}$ Physiology Research Center, Kerman University of Medical Sciences, Kerman, Iran. ${ }^{5}$ Air Pollution and Respiratory Diseases Research Center, Ahvaz Jundishapur University of Medical Sciences, Ahvaz, Iran. ${ }^{6}$ Environmental Technologies Research Center (ETRC), Ahvaz Jundishapur University of Medical Sciences, Ahvaz, Iran. ${ }^{7}$ Department of Environmental Health Engineering, School of Public Health, Tehran University of Medical Sciences, Tehran, Iran. ${ }^{8}$ Center for Air Pollution Research and Department of Research Methodology and Data Analysis, Institute for Environmental Research (IER), Tehran University of Medical Sciences, Tehran, Iran. ${ }^{9}$ Department of Epidemiology and Biostatistics, School of Public Health, Kerman University of Medical Sciences, Kerman, Iran.

\section{Received: 15 February 2017 Accepted: 9 March 2018}

Published online: 20 March 2018

\section{References}

1. Liu G-J, Fu E-J, Wang Y-J, Zhang K-F, Han B-P, ARROWSMITH C. A framework of environmental modelling and information sharing for urban air pollution control and management. J China Univ Min Technol. 2007;17(2):172-8.

2. Yamamoto SS, Phalkey R, Malik AA. A systematic review of air pollution as a risk factor for cardiovascular disease in South Asia: limited evidence from India and Pakistan. Int J Hyg Environ Health. 2014;217(2):133-44.

3. Atkinson RW, Analitis A, Samoli E, Fuller GW, Green DC, Mudway IS, et al. Short-term exposure to traffic-related air pollution and daily mortality in London, UK. J Expo Sci Environ Epidemiol. 2016;26(2):125.

4. Lopez-Villarrubia E, Ballester F, Iniguez C, Peral N. Air pollution and mortality in the Canary Islands: a time-series analysis. Environ Health. 2010;9(1):8.

5. Peng RD, Dominici F, Pastor-Barriuso R, Zeger SL, Samet JM. Seasonal analyses of air pollution and mortality in 100 US cities. Am J Epidemiol. 2005;161(6):585-94.

6. Hansel NN, McCormack MC, Kim V. The effects of air pollution and temperature on COPD. COPD: J Chron Obstruct Pulmon Dis. 2016;13(3):372-9.

7. Qorbani M, Yunesian M. Study designs in air pollution epidemiology. Iranian. Journal of Epidemiology. 2010;5(4):44-52.

8. World Health Organization. Ambient (outdoor) air quality and health 2016[Updated September 2016, Cited 16, Dec 2016]; Available from: http://www.who.int/mediacentre/factsheets/fs313/en/

9. Dadbakhsh M, Khanjani N, Bahrampour A. Death from respiratory diseases and air pollutants in shiraz, Iran (2006-2012). Journal of Environment Pollution and Human Health. 2015;3(1):4-11.

10. Hosseinpoor AR, Forouzanfar MH, Yunesian M, Asghari F, Naieni KH, Farhood D. Air pollution and hospitalization due to angina pectoris in Tehran, Iran: a time-series study. Environ Res. 2005;99(1):126-31.

11. Khalilzadeh S, Khalilzadeh Z, Emami H, Masjedi MR. The relation between air pollution and cardiorespiratory admissions in Tehran. Tanaffos. 2009;8(1):35-40.

12. Dominici F, McDermott A, Zeger SL, Samet JM. On the use of generalized additive models in time-series studies of air pollution and health. Am J Epidemiol. 2002;156(3):193-203.

13. Guisan A, Edwards TC, Hastie T. Generalized linear and generalized additive models in studies of species distributions: setting the scene. Ecol Model. 2002;157(2):89-100.
14. Wang $\mathrm{Y}$, Pham H. Analyzing the effects of air pollution and mortality by generalized additive models with robust principal components. International Journal of System Assurance Engineering and Management. 2011;2(3):253-9.

15. Spix C, Heinrich J, Dockery D, Schwartz J, Völksch G, Schwinkowski K, et al. Air pollution and daily mortality in Erfurt, East Germany, 1980-1989. Environ Health Perspect. 1993;101(6):518.

16. Khanjani N, Ranadeh Kalankesh L, Mansouri F. Air pollution and respiratory deaths in Kerman, Iran (from 2006 till 2010). Iranian. Journal of Epidemiology. 2012;8(3):58-65.

17. Bhaskaran K, Gasparrini A, Hajat S, Smeeth L, Armstrong B. Time series regression studies in environmental epidemiology. Int J Epidemiol. 2013; 42(4):1187-95

18. Shahi AM, Omraninava A, Goli M, Soheilarezoomand HR, Mirzaei N. The effects of air pollution on cardiovascular and respiratory causes of emergency admission. EMERGENCY-an academic. Emerg Med J. 2014:2(3):107-14.

19. Cesar ACG, Carvalho Jr JA, Nascimento LFC. Association between NOx exposure and deaths caused by respiratory diseases in a medium-sized Brazilian city. Braz J Med Biol Res. 2015;48(12):1130-5.

20. Masjedi M, Jamaati H, Dokohaki P, Ahmad-zade Z, Ghavam M, Agin K. The association of air pollution with acute heart attacks and respiratory. The quarterly journal of School of Medicine, Shahid Beheshti University of Medical Sciences (research on Medicine). 2001;25:25-33.

21. Zhang F, Li L, Krafft T, LV J, Wang W, Pei D. Study on the association between ambient air pollution and daily cardiovascular and respiratory mortality in an urban district of Beijing. Int J Environ Res Public Health. 2011;8(6):2109-23.

22. Sun J, Barnes AJ, He D, Wang M, Wang J. Systematic review and meta-analysis of the association between ambient nitrogen dioxide and respiratory disease in China. Int J Environ Res Public Health. 2017;14(6):646.

23. Yang $Y, C a o ~ Y, L i ~ W, ~ L i ~ R$, Wang $M$, Wu Z, et al. Multi-site time series analysis of acute effects of multiple air pollutants on respiratory mortality: a populationbased study in Beijing, China. Sci Total Environ. 2015;508:178-87.

24. Hansell A, Ghosh RE, Blangiardo M, Perkins C, Vienneau D, Goffe K, et al. Historic air pollution exposure and long-term mortality risks in England and Wales: prospective longitudinal cohort study. Thorax. 2016;71(4):330-8.

25. Mansourian M, Javanmard SH, Poursafa P, Kelishadi R. Air pollution and hospitalization for respiratory diseases among children in Esfahan, Iran. Ghana medical journal. 2010;44(4):138-43.

26. Liang W-M, Wei H-Y, Kuo H-W. Association between daily mortality from respiratory and cardiovascular diseases and air pollution in Taiwan. Environ Res. 2009;109(1):51-8.

27. Rezaei S, Khanjani N, Mohammadi SS, Darabi FZ. The effect of air pollution on respiratory disease visits to the emergency department in Kerman, Iran. Journal of Health \& Development. 2016;4(4):306-3014.

28. Hassanvand MS, Naddafi K, Faridi S, Arhami M, Nabizadeh R, Sowlat MH, et al. Indoor/outdoor relationships of PM 10, PM 2.5, and PM 1 mass concentrations and their water-soluble ions in a retirement home and a school dormitory. Atmos Environ. 2014:82:375-82.

29. Mazaheri Tehrani A, Karamali F, Chimehi E. Evaluation of 5 air criteria pollutants Tehran, Iran. International archives of. Health Sciences. 2015;2(3):95-100.

30. Wang Y, Zhuang G, Tang A, Yuan H, Sun Y, Chen S, et al. The ion chemistry and the source of PM 2.5 aerosol in Beijing. Atmos Environ. 2005;39(21): $3771-84$.

31. Dadbakhsh M, Khanjani N, Bahrampour A. Death from cardiovascular diseases and air pollution in shiraz, Iran (march 2006-march 2012). Journal of epidemiology and Preventive Medicine. 2016;2(2):114.

32. Dastoorpoor M, Idani E, Khanjani N, Goudarzi G, Bahrampour A. Relationship between air pollution, weather, traffic, and traffic-related mortality. Trauma Monthly. 2016;21(4):e37585.

33. Hashemi SY, Khanjani N. Air pollution and cardiovascular hospital admissions in Kerman, Iran. Journal of Heart and Cardiology. 2016;2(2):1-6.

34. Hashemi SY, Khanjani N, Soltaninejad Y, Momenzadeh R. Air Pollution and Cardiovascular mortality in Kerman from 2006 to 2011. American Journal of Cardiovascular Disease Research. 2014;2(2):27-30.

35. Vahedian M, Khanjani N, Mirzaee M, Koolivand A. Associations of short-term exposure to air pollution with respiratory hospital admissions in arak, Iran. J Environ Health Sci Eng. 2017;15(1):17.

36. Vahedian M, Khanjani N, Mirzaee M, Koolivand A. Ambient air pollution and daily hospital admissions for cardiovascular diseases in arak, Iran. ARYA Atheroscler. 2017;13(3):117-34. 